Wright State University

CORE Scholar

Physics Faculty Publications

Physics

$7-1-2001$

\title{
Characterization of Near-Surface Traps in Semiconductors: GaN
}

David C. Look

Wright State University - Main Campus, david.look@wright.edu

Z-Q. Fang

Follow this and additional works at: https://corescholar.libraries.wright.edu/physics

Part of the Physics Commons

\section{Repository Citation}

Look, D. C., \& Fang, Z. (2001). Characterization of Near-Surface Traps in Semiconductors: GaN. Applied Physics Letters, 79 (1), 84-86.

https://corescholar.libraries.wright.edu/physics/68

This Article is brought to you for free and open access by the Physics at CORE Scholar. It has been accepted for inclusion in Physics Faculty Publications by an authorized administrator of CORE Scholar. For more information, please contact library-corescholar@wright.edu. 


\title{
Characterization of near-surface traps in semiconductors: GaN
}

\author{
D. C. Look ${ }^{\text {a) }}$ and Z.-Q. Fang \\ Semiconductor Research Center, Wright State University, Dayton, Ohio 45435
}

(Received 2 February 2001; accepted for publication 25 April 2001)

\begin{abstract}
We present a simple a criterion, based on deep-level transient spectroscopy peak heights $S\left(V_{r}\right)$ at two or more values of reverse bias $V_{r}$, to unequivocally determine whether or not a particular semiconductor trap is of bulk or near-surface nature. Moreover, we present an expression for $S\left(V_{r}\right)$ with fitting parameters $\phi_{B}$, the Schottky barrier height; $\delta$, the trap penetration depth; and $N_{T}$, the trap density. Application of the method to a thick, high-quality, epitaxial GaN layer, reveals two common traps which penetrate only $2700 \pm 300 \AA$ into the layer. (C) 2001 American Institute of Physics. [DOI: 10.1063/1.1379982]
\end{abstract}

Electron and hole traps in semiconductor materials are of high importance because they often affect the dynamical properties of electronic and photonic devices. For example, response times in GaAs field-effect transistors can be degraded by traps near the substrate, and breakdown voltages can be reduced by traps near the surface. Furthermore, lowfrequency noise can be generated by both bulk and surface traps. Thus, many laboratories have established techniques to study traps, and the most common of these is the capacitance version of deep-level transient spectroscopy (DLTS). ${ }^{1,2}$ DLTS experiments, in their simplest form, yield "apparent" values of trap energy, concentration, and capture cross section; however, the determination of the "true" values often requires more complicated experiments and analyses. ${ }^{2,3}$ For example, it is well known that the usual analysis gives an artificially low value of trap concentration (the " $\lambda$ effect"), because it is based on the assumption that the forward bias pulse fills all traps in the depletion region, whereas some of the deeper traps are not empty before the pulse. ${ }^{2-4}$ At higher reverse biases the apparent trap concentration will increase and, in fact, an analysis of signal height versus forward bias voltage can often be used to determine the true trap concentration, if it is constant with depth. ${ }^{5}$ Sometimes, however, the apparent trap concentration decreases as reverse bias increases (becomes more negative), which is the opposite of what is expected. ${ }^{6}$ In this letter, we show that such behavior is a direct proof of a near-surface trap, i.e., one which does not exist beyond the depth $\delta<w_{r}-\lambda$, where $w_{r}$ is the depletion depth at reverse bias $V_{r}$, and $\lambda$ is a length which is dependent on trap energy and donor concentration. For such traps, we also show that the ratio of any two peak heights, measured at two different reverse-bias voltages, is a constant dependent only on the two voltages, and is independent of forward bias voltage and any trap parameters, including energy or penetration depth $\delta$ (from the surface). The analysis developed here is used to establish the bulk or near-surface nature of traps in a recently introduced form of $\mathrm{GaN}$ grown by hydride vapor phase epitaxy (HVPE) ${ }^{7-11}$

The capacitative DLTS experiment is based on observing a change in capacitance $\Delta C$ as a result of a forward-bias pulse which fills traps and thus changes their charge states.

a)Electronic mail: david.look@wpafb.af.mil
Consider a donor-type trap of concentration $N_{T}$ in a Schottky-barrier diode formed on an $n$-type semiconductor material with net shallow donor concentration $N_{D}^{\text {net }}=N_{D}$ $-N_{A}$. At a reverse bias $V_{r}$ (negative value), the diode will have a capacitance $C=\epsilon A / w_{r}$, where $\epsilon$ is the dielectric constant, $A$ is the diode area, and

$$
w_{r}=\sqrt{\frac{2 \epsilon\left[\phi_{B}-V_{r}-E_{C}(T) / e-k T / e\right]}{e N_{D}^{\text {net }}}},
$$

where $\phi_{B}$ is the Schottky-barrier height, $k$ is Boltzmann's constant, and $E_{C}(T)$ is the position of the conduction band with respect to the Fermi level at depth $z=\infty{ }^{2,3}$ In the nondegenerate limit, $E_{C}(T)=k T \ln \left(N_{C} / n\right)$, where $N_{C}$ is the effective conduction-band density of states. Typically, $E_{C}(T) / e$ and $k T / e$ are small compared to $\phi_{B}-V_{r}$.

Consider the band diagram of Fig. 1, which shows conduction-band and trap energies as a function of depth $z$, for reverse bias $V_{r}=-8 \mathrm{~V}$, and forward bias $V_{f}=0 \mathrm{~V}$. Here the Fermi energy is set at $E=0$. By definition, all of the shallow donors in the depletion region, $z=0$ to $w_{r}$, are ionized, i.e., positively charged. However, a typical trap of in-

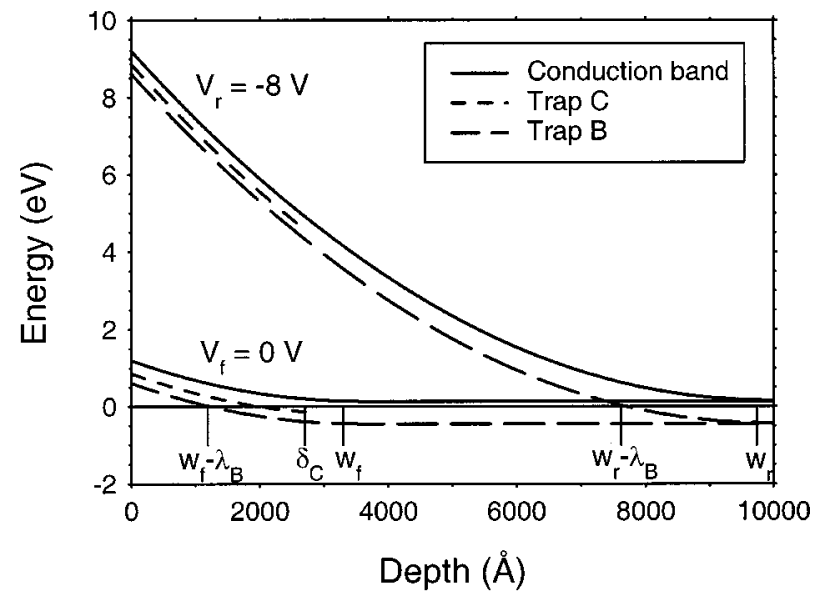

FIG. 1. Conduction-band, trap-C, and trap-B energies vs depth for $V_{r}$ $=-8 \mathrm{~V}$, and $V_{f}=0 \mathrm{~V}$, obtained by solving the Poisson equation with the following parameters: $T=300 \mathrm{~K} ; m^{*}=0.22 m_{0} ; \epsilon=9.5 \epsilon_{0} ; \phi_{B}=1.2 \mathrm{~V} ; N_{D}$ $=1.5 \times 10^{16} \mathrm{~cm}^{-3} ; N_{A}=5 \times 10^{15} \mathrm{~cm}^{-3} ; E_{B}=0.59 \mathrm{eV} ;$ and $E_{C}=0.34 \mathrm{eV}$. The Fermi energy is set at $E=0$. 
terest will have energy $E_{T}>E_{D}$, and thus its energy will cross the Fermi energy at a depth $z<w_{r}$, in fact at $z=w_{r}$ $-\lambda$, where

$$
\lambda=\sqrt{\frac{2 \epsilon\left[E_{T}-E_{C}(T)\right]}{e^{2} N_{D}^{\text {net }}}} .
$$

Therefore, this particular trap will be ionized only from $z$ $=0$ to $w_{r}-\lambda$, and a forward-bias pulse will be able to fill no more than a fraction $\left(w_{r}-\lambda\right) / w_{r}$ of the traps, since the rest are already filled before the pulse. Immediately after the forward-bias pulse, the reverse-bias depletion depth $w_{r}$ will increase by $\Delta w$, in order to accommodate the increased negative charge in the depletion region. By solving Poisson's equation in the depletion approximation, we can show that ${ }^{2}$

$$
\int_{w_{f}-\lambda}^{w_{r}-\lambda} z N_{T} d z=\int_{w_{r}}^{w_{r}+\Delta w} z N_{D}^{\mathrm{net}} d z \approx \frac{N_{D}^{\mathrm{net}}}{2} 2 w_{r} \Delta w,
$$

where it has also been assumed that $\Delta w \ll w_{r}$ (true if $N_{T}$ $\left.\ll N_{D}\right)$, and that $N_{D}$ is uniform in the region $w_{r}<z<w_{r}$ $+\Delta w$. The usual formula for the $\lambda$ effect is developed by also assuming that $N_{T}$ is uniform in the region $w_{f}-\lambda<z$ $<w_{r}-\lambda$, and then solving ${ }^{2}$ for the DLTS signal, $S$ $\equiv-\Delta C / C$ :

$$
\begin{aligned}
S & =-\frac{\Delta C}{C}=\frac{\Delta w}{w_{r}}=\frac{N_{T}}{2 N_{D}^{\text {net }}} \frac{\left(w_{r}-\lambda\right)^{2}-\left(w_{f}-\lambda\right)^{2}}{w_{r}^{2}} \\
& \equiv f_{\lambda} \frac{N_{T}}{2 N_{D}^{\text {net }}}, \quad \text { for } \delta>w_{r}-\lambda .
\end{aligned}
$$

Clearly $f_{\lambda}<1$, but it increases with $w_{r}$ (or as $V_{r}$ becomes more negative), eventually approaching unity ${ }^{5}$ for $w_{r} \gg w_{f}$ and $\lambda$. The trap-analysis program supplied with a typical commercial DLTS system, such as our BioRad DL4600, is based on the assumption $f_{\lambda}=1$, so that the trap concentration will always be underestimated.

We now consider the situation in which a particular trap is cut off at a depth $\delta<w_{r}-\lambda$; i.e., we assume that the trap exists only in the region $0<z<\delta$, as illustrated for trap $\mathrm{C}$ in Fig. 1. Then the upper limit to the integral on the left-hand side of Eq. (3) is $\delta$, not $w_{r}-\lambda$, and, as long as $\delta>w_{f}-\lambda$, Eq. (4) becomes

$$
\begin{aligned}
S= & -\frac{\Delta C}{C}=\frac{\Delta w}{w_{r}}=\frac{N_{T}}{2 N_{D}^{\text {net }}} \frac{\delta^{2}-\left(w_{f}-\lambda\right)^{2}}{w_{r}^{2}} \equiv f_{\lambda \delta} \frac{N_{T}}{2 N_{D}^{\text {net }}}, \\
& \text { for } \quad w_{f}-\lambda<\delta<w_{r}-\lambda .
\end{aligned}
$$

In this case, $S$ decreases with increasing $w_{r}$ (more negative $V_{r}$ ), the opposite of the expected behavior. By comparing Eqs. (4) and (5), it is clear that a decrease in $S$ with more negative $V_{r}$ is a proof that $\delta<w_{r}-\lambda$. But Eq. (5) also leads to a very simple relationship between signals $S(V)$ at two different reverse biases, $V_{r 1}$ and $V_{r 2}$ :

$$
\begin{aligned}
\frac{S\left(V_{r 1}\right)}{S\left(V_{r 2}\right)}= & \frac{\phi_{B}-V_{r 2}-E_{C}(T) / e-k T / e}{\phi_{B}-V_{r 1}-E_{C}(T) / e-k T / e} \approx \frac{\phi_{B}-V_{r 2}}{\phi_{B}-V_{r 1}}, \\
& \text { for } w_{f}-\lambda<\delta<w_{r}-\lambda .
\end{aligned}
$$

Note that the right-hand side of Eq. (6) is independent of any trap parameters or the forward bias voltage, and moreover is completely determined, since $\phi_{B}$ can be calculated indepen-

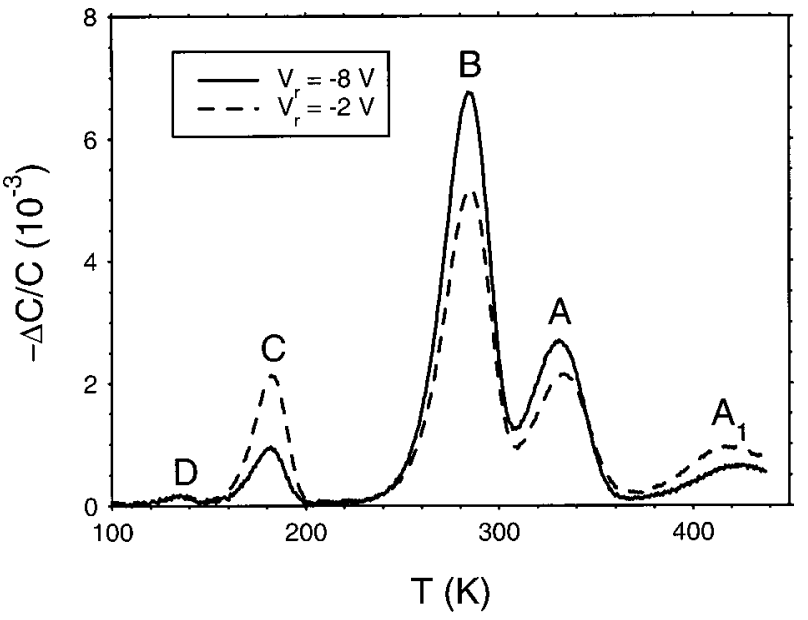

FIG. 2. DLTS spectra at reverse-bias voltages of -8 and $-2 \mathrm{~V}$, respectively. The other conditions are: forward-bias voltage of $0 \mathrm{~V}$; filling pulse length of $1 \mathrm{~ms}$; and rate window of $4 / \mathrm{s}$.

dently from a $1 / C^{2}$ vs $V_{r}$ plot. Also, note that $N_{T}$ does not have to be uniform in the region of its existence, for Eq. (6) to hold. That is, the numerator of Eq. (5) could be left in integral form, and Eq. (6) would still be valid. This observation is important for ion-implanted samples, for example. We will refer to the above phenomenon, defined by Eqs. (5) and (6), as the " $\lambda \delta$ effect," to contrast it with the " $\lambda$ effect," Eq. (4), which holds only for bulk traps.

If $N_{T}$ is uniform, then the trap penetration depth $\delta$ can be determined by varying $V_{r}$ over a wide enough range to include the conditions $\delta<w_{r}-\lambda$, and $\delta>w_{r}-\lambda$, and then fitting a normalized $S\left(V_{r}\right)$ to Eqs. (4) and (5). It is usually convenient to normalize to the most negative $V_{r}$ used in the experiment. In our case, we have plotted the function $S$ $(-8 V) / S\left(V_{r}\right)$ vs $V_{r}$, because then Eq. (6) predicts a straight line as long as $\delta<w_{r}-\lambda$, and a rapid break from the straight line as soon as $\delta>w_{r}-\lambda$.

We apply the analysis developed here to a $300-\mu \mathrm{m}$-thick GaN layer grown by the HVPE technique and separated from its sapphire substrate. Recent optical, ${ }^{7-9}$ electrical, ${ }^{7,8,10,11}$ and structural ${ }^{8,9}$ measurements, including DLTS, ${ }^{11}$ have demonstrated the excellent quality of such material; e.g., one wafer has exhibited the highest $\mathrm{GaN}$ mobility ever measured $\left(\sim 1200 \mathrm{~cm}^{2} / \mathrm{V} \mathrm{s}\right.$ at $\left.300 \mathrm{~K}\right)$, and the lowest donor and acceptor concentrations, about $8 \times 10^{15}$ and $3 \times 10^{15} \mathrm{~cm}^{-3}$, respectively. ${ }^{10}$ However, the particular wafer studied here had $N_{D}^{\text {net }}=1 \times 10^{16} \mathrm{~cm}^{-3}$, from $C-V$ measurements on the same Schottky-barrier diode (SBD) as that used for DLTS. The previous DLTS study of this material ${ }^{11}$ identified five common traps on Ga-face SBDs, with energies as follows: $\mathrm{A}_{1}(1.04 \mathrm{eV}), \mathrm{A}(0.66 \mathrm{eV}), \mathrm{B}(0.59 \mathrm{eV}), \mathrm{C}(0.34 \mathrm{eV})$, and $\mathrm{D}(0.24 \mathrm{eV})$. (However, that study ${ }^{11}$ did not determine concentrations accurately, because the $\lambda \delta$ effect was not taken into account). Each of these traps has been seen in earlier DLTS studies of GaN grown by various epitaxial techniques or subjected to certain types of processing. ${ }^{12,13} \mathrm{~A}$ trap $\mathrm{B}^{\prime}$ $(0.46 \mathrm{eV})$ was also seen,${ }^{11}$ but will not be further discussed here. The sample studied in this work also contains traps $A_{1}$, A, B, C, and D, but not $\mathrm{B}^{\prime}$, as shown in Fig. 2. Traps A, B, and $\mathrm{D}$ basically display an increasing $-\Delta C / C$ with more negative reverse bias, which is normal; however, traps $A_{1}$ 


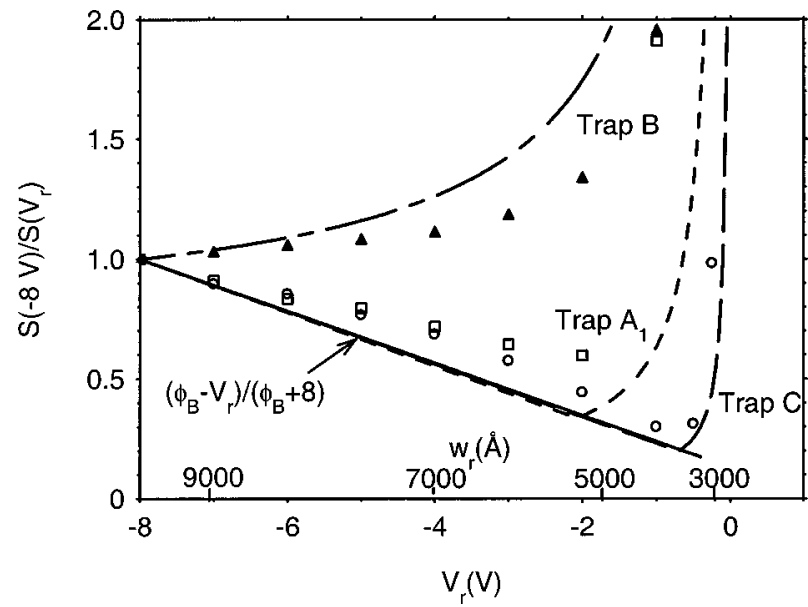

FIG. 3. The ratio $S(-8 \mathrm{~V}) / S\left(V_{r}\right)$ vs $V_{r}$ for traps B (triangles), C (circles), and $\mathrm{A}_{1}$ (squares), where $S \equiv-\Delta C / C$. Also plotted on the abscissa are the values of depletion length $w_{r}$ at given values of $V_{r}$. The dashed and chained lines are theoretical fits. The solid line is the function $\left(\phi_{B}-V_{r}\right) /\left(\phi_{B}+8\right)$.

and $\mathrm{C}$ have an opposite behavior. Thus, from Eqs. (4) and (5), traps A, B, and D are "bulk" in nature $\left(\delta>w_{r}-\lambda\right)$, and $\mathrm{A}_{1}$ and $\mathrm{C}$ are "near-surface" $\left(\delta<w_{r}-\lambda\right)$. These assertions are verified by plotting the ratio $S(-8 V) / S\left(V_{r}\right)$ vs $V_{r}$ for traps $\mathrm{B}, \mathrm{C}$, and $\mathrm{A}_{1}$, in Fig. 3. It is seen that trap B is a normal bulk trap, since the ratio continuously increases as $V_{r}$ becomes less negative. By bulk, in this case, we mean that $\delta_{\mathrm{B}}>w_{r}(-8 \mathrm{~V})-\lambda_{\mathrm{B}}=7560 \AA$, where $w_{r}$ and $\lambda$ are evaluated at $E_{T}=0.59 \mathrm{eV}$, and $T_{\text {peak }}=295 \mathrm{~K}$. These parameters, along with $\phi_{B}=1.2 \mathrm{~V}$ and $N_{D}^{\text {net }}=1 \times 10^{16} \mathrm{~cm}^{-3}$, are then used in Eq. (4) to produce the chained line in Fig. 3. The fit must be considered satisfactory considering that there are no arbitrary fitting parameters. Interestingly, a much better fit can be obtained with a smaller value of $E_{T}$, which suggests that the $0.59 \mathrm{eV}$ obtained from an Arrhenius analysis $\left(E_{\mathrm{Arr}}=E_{T}\right.$ $+E_{\sigma}$ ) may contain a capture-cross-section term, $E_{\sigma}$.

In contrast, for traps $\mathrm{C}$ and $\mathrm{A}_{1}$, the ratio $S$ $(-8 \mathrm{~V}) / S\left(V_{r}\right)$ initially decreases as $V_{r}$ becomes less negative, proving immediately that $\delta_{C}<8120 \AA$, and $\delta_{\mathrm{A} 1}$ $<6770 \AA$. However, the ratio in each case begins to rapidly increase at a particular value of $V_{r}$, allowing us to actually calculate $\delta$, by fitting to Eqs. (4) and (5). Here, the only fitting parameter is $\delta$, and a value $\delta=2700 \AA$ produces satisfactory fits for both traps $\mathrm{C}$ and $\mathrm{A}_{1}$. Furthermore, in the region $\delta<w_{r}-\lambda$, the data reasonably follow the function $\left(\phi_{B}-V_{r}\right) /\left(\phi_{B}+8\right)$, shown as a solid line. It is perhaps more significant that the ratios for traps $\mathrm{C}$ and $\mathrm{A}_{1}$ are nearly equal to each other, until the curves begin to break off. This independence of trap parameters is indeed predicted by Eq. (6), for $\delta<w_{r}-\lambda$. Thus, it is clearly evident that traps $\mathrm{C}$ and $\mathrm{A}_{1}$ extend only about $2700 \AA \pm 10 \%$ into the GaN layer. Finally, Eqs. (4) and (5) can now be used to obtain accurate values of $N_{T}$, which are $2.4 \times 10^{14}, 4.5 \times 10^{14}$, and $2.1 \times 10^{14} \mathrm{~cm}^{-3}$, for traps $\mathrm{B}, \mathrm{C}$, and $\mathrm{A}_{1}$, respectively. Note that the usual analysis would have calculated the concentrations from the high-reverse-bias $(-8 \mathrm{~V})$ data of Fig. 2, using the equation $N_{T}=2 N_{D}(-\Delta C / C)$, giving $1.3 \times 10^{14}, 1.9 \times 10^{13}$, and 1.3 $\times 10^{13} \mathrm{~cm}^{-3}$, respectively. Thus, the usual analysis is off by more than an order of magnitude for traps $\mathrm{C}$ and $\mathrm{A}_{1}$, and it is critical to take account of the $\lambda \delta$ effect.

Although we do not yet know the origin of trap C, it is probably defect-related, because, e.g., it seems to be generated during ion-beam etching ${ }^{11}$ and sputtering. ${ }^{13}$ Such processes would be expected to produce damage only up to a few $1000 \AA{ }^{14}$ as observed here. Trap $\mathrm{A}_{1}$ also is likely to be defect-related, because it is quite similar to a trap produced by high-energy electron irradiation, ${ }^{12}$ heavy-ion irradiation, ${ }^{15}$ and electron-beam deposition. ${ }^{16}$ Finally, traps $\mathrm{C}$ and $\mathrm{A}_{1}$ may be related to each other, since they have similar depths of penetration $(2700 \AA)$. These matters are important to understand, and further research will be carried out.

In conclusion, we have developed a very robust criterion to determine whether traps are distributed throughout the depletion region (bulk-like), or are concentrated near the surface. This criterion and the associated quantitative analysis have been applied to GaN grown by the HVPE process, and two out of five traps in this case are shown to be of nearsurface origin; furthermore, their concentrations are accurately determined. The analysis should also be applicable to most other semiconductor materials, and can explain some of the literature data formerly described as anomalous.

The authors wish to thank S. S. Park and K. Y. Lee, of Samsung Advanced Institute of Technology, for providing the GaN samples; P. Visconti, C.-Z. Lu, D.-F. Wang, and H. Morkoç, of Virginia Commonwealth University, for fabricating the Schottky diodes and for helpful discussions; and L. Callahan, of the Air Force Research Laboratory (AFRL), for bonding the diodes. The authors were supported under AFOSR Contract No. F49620-00-1-0347.

${ }^{1}$ D. V. Lang, J. Appl. Phys. 45, 3023 (1974).

${ }^{2}$ D. C. Look, Electrical Characterization of GaAs Materials and Devices (Wiley, New York, 1989), Chap. 4.

${ }^{3}$ D. C. Look and J. R. Sizelove, J. Appl. Phys. 78, 2848 (1995).

${ }^{4}$ Y. Zohta and M. O. Watanabe, J. Appl. Phys. 53, 1809 (1982).

${ }^{5}$ D. C. Look, Z.-Q. Fang, and J. R. Sizelove, J. Appl. Phys. 77, 1407 (1995)

${ }^{6}$ A. Y. Polyakov, A. S. Usikov, B. Theys, N. B. Smirnov, A. V. Govorkov, F. Jomard, N. M. Shmidt, and W. V. Lundin, Solid-State Electron. 44, 1971 (2000)

${ }^{7}$ D. C. Reynolds, D. C. Look, B. Jogai, A. W. Saxler, S. S. Park, and J. Y. Han, Appl. Phys. Lett. 77, 2879 (2000).

${ }^{8}$ F. Yun, M. A. Reshchikov, K. M. Jones, P. Visconti, H. Morkoç, S. S. Park, and K. Y. Lee, Solid-State Electron. 44, 2225 (2000).

${ }^{9}$ E. Oh, S. K. Lee, S. S. Park, K. Y. Lee, I. J. Song, and J. Y. Han, Appl. Phys. Lett. 78, 273 (2001).

${ }^{10}$ A. Saxler, D. C. Look, S. Elhamri, J. R. Sizelove, W. C. Mitchel, C. M. Sung, S. S. Park, and K. Y. Lee, Appl. Phys. Lett. 78, 1873 (2001).

${ }^{11}$ Z.-Q. Fang, D. C. Look, P. Visconti, D.-F. Wang, C.-Z. Lu, F. Yun, H. Morkoç, S. S. Park, and K. Y. Lee, Appl. Phys. Lett. 78, 2178 (2001).

${ }^{12}$ Z.-Q. Fang, L. Polenta, J. W. Hemsky, and D. C. Look, in "2000 International Semiconducting and Insulating Materials Conference," edited by C. Jagadish and N. J. Welham (IEEE, Piscataway, N.J., 2000), p. 35.

${ }^{13}$ F. D. Auret, S. A. Goodman, F. K. Koschnick, J.-M. Spaeth, B. Beaumont, and P. Gibart, Appl. Phys. Lett. 74, 2173 (1999).

${ }^{14}$ R. Cheung, R. J. Reeves, S. A. Brown, E. van der Drift, and M. Kamp, J. Appl. Phys. 88, 7110 (2000).

${ }^{15}$ F. D. Auret, S. A. Goodman, F. K. Koschnick, J.-M. Spaeth, B. Beaumont, and P. Gibart, Appl. Phys. Lett. 73, 3745 (1998).

${ }^{16}$ F. D. Auret, S. A. Goodman, G. Myburg, F. K. Koschnick, J.-M. Spaeth, B. Beaumont, and P. Gibart, Physica B 273-274, 84 (1999). 